\title{
IMPLEMENTACIÓN DE LABORATORIOS VIRTUALES COMO ALTERNATIVA PARA ESTIMULAR LAS ACTIVIDADES ACADÉMICAS EN EL AULA DE CLASE
}

\author{
IMPLEMENTATION OF VIRTUAL LABORATORIES AS AN ALTERNATIVE TO \\ STIMULATE THE ACADEMIC ACTIVITIES IN THE CLASSROOM
}

\author{
Carlos Alberto Ramírez Vanegas로 Carlos Rodríguez Varela², Germán Correa Vélez L³ \\ Recibido para publicación: 5 de abril 2017 - Aceptado para publicación: 31 de mayo 2017
}

\section{RESUMEN}

Actualmente la formación que es impartida a los estudiantes de ingeniería tiene un alto contenido teórico el cual la mayoría de las veces no se logra mostrar a través de la práctica por la falta de recursos para la implementación de laboratorios. Lo anterior conlleva a que los estudiantes no refuercen sus conocimientos a través de la ejemplificación. De acuerdo a lo expuesto se propone que el contenido teórico sea verificado a través de softwares, esto no solamente ayuda al estudiante a mejorar su aprendizaje de una manera sencilla sino también es una buena simulación del entorno real minimizando costos y aportándole al estudiante de ingeniería una herramienta más que el sector laboral le demandará. En el presente trabajo se muestra la implementación de dos softwares, uno para ingeniería eléctrica y otro para ingeniería industrial. El primero ISIS PROTEUS para la implementación de circuitos electrónicos y eléctricos y el segundo PROMODEL para ejemplificación de casos de producción, los cuales de una manera económica y sencilla muestran de una forma aplicada conceptos y que además permite hacer variaciones en las variables lo que en laboratorios físicos es más tedioso y costoso por el uso de materiales.

Palabras clave: Laboratorio virtual, software, educación.

\begin{abstract}
The above leads to students not reinforce their knowledge through exemplification. According to the foregoing it is proposed that the theoretical content is verified through software, this not only helps students to improve their learning in a simple way but also is a good simulation of the real environment by minimizing costs and providing to the engineering student a tool that will require the employment sector. In this paper is shown the implementation of two softwares, one for electrical engineering and other for industrial engineering. The first MATLAB for implementing electrical circuitry and the second PROMODEL for production modeling, which in an economical and easily applied concepts show an applied way concepts, and it also allow variations in the physical variables which is tedious in the laboratories and expensive.
\end{abstract}

Key word: Virtual laboratory, software, education

\footnotetext{
1 Departamento de matemáticas, Universidad Tecnológica de Pereira, Pereira, Colombia caramirez@utp.edu.co

2 Departamento de matemáticas, Universidad Tecnológica de Pereira, Pereira, Colombia car@utp.edu.co

${ }^{3}$ Departamento de matemáticas, Universidad Tecnológica de Pereira, Pereira, Colombia gecove@utp.edu.co
} 


\section{INTRODUCCIÓN}

En los últimos años el mundo ha vivido tiempos de cambio y de innovaciones en todos los ámbitos gracias al avance de la tecnología. La educación como actividad humana fundamental tampoco es ajena a estos movimientos y es así como hoy se ven herramientas tecnológicas dentro de las aulas de clase, desde un video-beam y un computador para realizar presentaciones en Power Point como alternativa para dictar las clases, hasta micro-celdas de manufactura que permiten un acercamiento a los estudiantes a un contexto real y les ayudan a interiorizar de una mejor manera los conceptos teóricos que son impartidos en las clases.

Los medios educativos tecnológicos los cuales son definidos como aquellos elementos materiales cuya función estriba en facilitar la comunicación que se establece entre educadores y educandos (Colom, Salinas, \& Sureda, 1988) son fundamentales para generarle al estudiante un aprendizaje basado en un modelo didáctico el cual no se preocupa solo del contenido de la enseñanza sino también de la forma como los conceptos son enseñados buscando siempre la unión de la teoría y la práctica, entendiendo que estas dos se complementan y mejoran considerablemente el proceso de enseñanzaaprendizaje (Aguilar \& Viniegra, 2003).

Cabe resaltar que para tener altas tecnologías en los lugares de enseñanza se deben recurrir a altos costos los cuales posiblemente no están al alcance de universidades pertenecientes a países en vía de desarrollo y es ahí donde el presente trabajo cobra importancia, puesto que propone el uso de tecnologías asequibles y fáciles de manejar como alternativa para llevar a cabo ese componente práctico que tanto necesitan los estudiantes para reforzar sus conocimientos y que muchas veces por falta de recursos deja de hacerse.

El trabajo propone la utilización de dos softwares que permiten unir el conocimiento y la acción en temas de producción, logística y circuitos, para que así el alumno pueda tener una mayor comprensión de los mismos. El primero es ProModel el cual es un software de simulación, que permite a un usuario construir modelos y escenarios, el cual le da la libertad al estudiante de modificar las variables que influyen en un proceso, con el fin de que pueda identificar los diferentes conceptos del área de producción, reconociendo incluso problemas y aciertos del modelo, y con base en esto proponer mejoras las cuales puede ser verificadas por el mismo.

\section{CASO PROMODEL}

\subsection{DESCRIPCION DEL PROMODEL}

ProModel es un software que permite simular cualquier tipo de sistemas de manufactura, logística, manejo de materiales, etc. Este permite además simular bandas de transporte, grúas viajeras, ensamble, corte, talleres, logística, entre otros (ProModel).

La simulación a través de esta herramienta le brinda al estudiante la oportunidad de analizar procesos y mejorarlos, permitiéndole conocer los impactos de los cambios en los procesos sin necesidad de llevarlos a cabo en la realidad.

Cabe resaltar la facilidad de manejo que tiene ProModel, el cual no requiere programación, aunque sí lo permite.

Dentro de los temas del área de producción y logística que se pueden simular a través del ProModel se encuentra el justo a tiempo, teoría de restricciones, sistemas de empujar, sistema de jalar, los diferentes modelos clásicos de producción (lineal, por proyectos, etc.), funcionamiento de la cadena logística, teoría de colas, etc.

Cuando se simula cualquiera de los anteriores temas, ProModel brinda ciertas medidas del proceso las cuales son analizadas para tomar decisiones frente al mismo. Por ejemplo, para el caso de la simulación de un proceso de producción, ProModel permite medir el uso de la capacidad de las estaciones de trabajo, del tiempo de las unidades en el sistema (throughput time), del trabajo que queda en proceso al terminar la jornada de trabajo (work in process), entre otras. A partir de estas medidas es posible incluso aplicar principio de manufactura que se hayan aprendido en el salón de clase, por ejemplo la ley de Little (Spearman \& Hopp, 1996) la cual permite determinar la tasa de productividad (production rate: $\mathrm{PR}$ ) a través del throughput time (TT) y el work in process (WIP) como lo muestra la Ec. 1

$$
\mathrm{PR}=\frac{\mathrm{WIP}}{\mathrm{TT}} P R=\frac{\text { WIP }}{T T} \quad \text { Ecuación } 1
$$




\subsection{EJEMPLO PROMODEL}

En el presente ejemplo se muestra como a través del modelamiento sencillo de un caso de producción los estudiantes pueden usar los conceptos aprendidos en los salones de clase para hacer un análisis de estas situaciones reales que se pueden modelar a través del ProModel.

Para este ejemplo se modelará un caso de producción de esqueletos, el cual se detalla a continuación:

En una fábrica de esqueletos, los cuellos y cuerpos llegan por bandas (una para cada parte, la longitud de cada una es de 20 metros, con una capacidad de 30) para unirse en la locación "pegado cuellos", en esta estación la operación dura 10,74 minutos en promedio con una desviación estándar de 2,33 y sale un producto en procesos denominado esq_cuello a la estación "pegado bolsillos" donde se demora 21,8 minutos en promedio con una desviación estándar de 5,71 , y sale como esq_cuello_bols, de allí va a la estación estampado excepto los productos esq cuello_bols que en su orden de llegada tienen múltiplo de cinco, las cuales pasan directamente a inspección (número 1, 2, 3, y 4 van a "estampado", las de clase número 5 van a inspección), en la estación "estampado" la operación se demora 11,38 minutos en promedio con una desviación de 3,22, de allí salen como "esq_cuello_bols_ estam" a inspección y luego fuera del sistema. La inspección demora 5,87 minutos en promedio con 5,47 de desviación estándar. Se sabe además que el $85 \%$ de los "esq_cuello_bols_estam" se aceptan y salen fuera del sistema, el resto pasan a mesa rechazos y luego pasan fuera del sistema.

Las llegadas tanto de los cuellos como los cuerpos son una cada 9 minutos.

Se desea saber el número de esq_cuello_bols que salieron del sistema, el número de esq cuello_bols_estam que salieron del sistema y los que fueron rechazados.

Las máquinas de coser de las estaciones "pegado bolsillo" y "pegado cuellos", y la máquina de estampados deben recibir mantenimiento cada dos horas, el mantenimiento dura 10 minutos en promedio con una desviación estándar de 0.2. Para esto hay un operario que trabaja de 8 a.m. a 6 p.m. de lunes a viernes. Con descansos así: 10:15 a.m. a 10:30 a.m. de 1:00 p.m. a 2:00 p.m. y de 4:00 p.m. a 4:15 p.m.

El cual tiene una red asignada para llevar a cabo sus tareas de mantenimiento (ir a "pegado bolsillos", "pegado cuellos" y "estampado", además la red está compuesta por otro nodo que es "descanso") su "home" es el nodo 1 que es descanso, es decir, cuando él no se encuentre haciendo mantenimiento estará en la locación "descanso". Las distancias de todos los caminos son de 20 metros.

A continuación, se encuentra el modelo grafico que representa las diferentes operaciones en el proceso de producción y el valor que tomaron las variables después de las 10 horas de corrida.

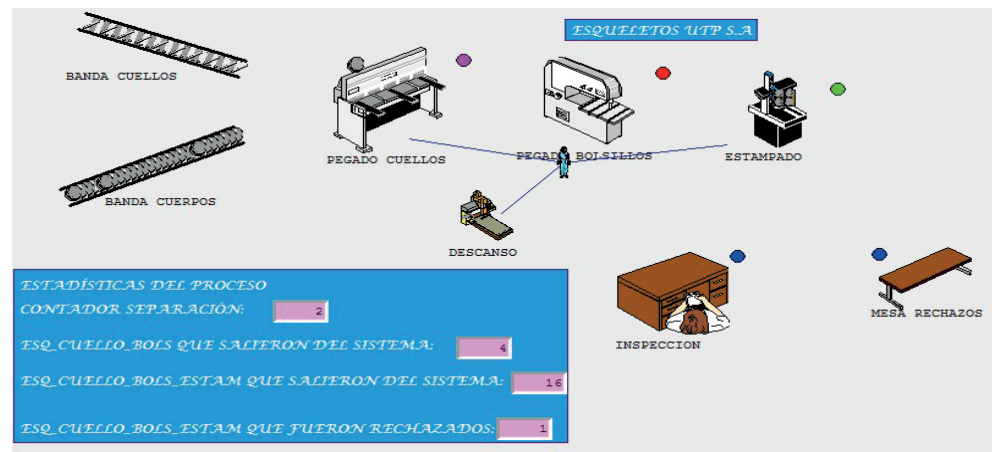

Figura 1. Gráfico del proceso de producción de esqueletos. Fuente: ProModel

A continuación se muestran algunos de los cuadros de resultados que genera ProModel con el fin de ejemplificar el tipo de análisis que se puede hacer basado en conceptos de producción:
De este cuadro se puede determinar el uso de la capacidad de las dos bandas que participan en el proceso de producción. Se observa que ambas bandas estuvieron totalmente vacías el 3,01\% 


\begin{tabular}{|c|c|c|c|c|c|c|c|}
\hline General & Locations & \multicolumn{2}{|c|}{ Location States Multi } & ocation States Single & \multicolumn{2}{|c|}{ Resources } & $\mathrm{H}$ \\
\hline \multicolumn{8}{|c|}{ EJEMPLO SINULACIÓN.MOD [Normal Run - Rep. 1] } \\
\hline \multicolumn{2}{|c|}{ Name } & $\begin{array}{c}\text { Scheduled } \\
\text { Time [HR] }\end{array}$ & \% Empty & $\%$ Part Dccupied & $\%$ Full & \multicolumn{2}{|c|}{ \% Down } \\
\hline \multicolumn{2}{|c|}{ BANDA CUELLOS } & 10,00 & 3,01 & 96,99 & 0,00 & & 0,0 \\
\hline \multicolumn{2}{|c|}{ BANDA CUERPOS } & 10,00 & 3,01 & 96,99 & 0,00 & & 0,00 \\
\hline
\end{tabular}

Figura 2. Ficha de resultados de las locaciones con capacidad múltiple.

Fuente: ProModel

del tiempo de simulación (10 horas), y el resto del tiempo estuvieron parcialmente ocupadas, es decir que no se utilizó la capacidad de las mismas en su totalidad. Lo anterior conlleva a que se preste atención al uso de la capacidad de las diferentes locaciones ya que puede ser que estén siendo subutilizadas.

De este cuadro se puede resaltar la utilización de la capacidad de cada una de las locaciones, donde se verifica que efectivamente el proceso no está trabajando al ritmo que podría rendir, lo cual tiene muchas consecuencias negativas, siendo una de ellas que se le debe atribuir un porcentaje alto de costo a cada producto fabricado cuando este podría ser menor si se usara efectivamente la capacidad de las locaciones produciendo mayor cantidad de productos. Aquí se puede incluir el concepto de rendimiento de producción a escala creciente el cual se da cuando al variar la cantidad utilizada de todos los factores, en una determinada proporción, la cantidad obtenida del producto varía en una proporción mayor; esto puede probarse variando los datos de entrada del modelo (como el tiempo de llegada de los cuellos y cuerpos, tiempo de operación en cada locación) para verificar los cambios en la utilización y determinar si se justifican estos cambios de acuerdo al aumento en la producción.

\begin{tabular}{|c|c|c|c|c|c|c|c|c|c|c|}
\hline & Locations & \multicolumn{2}{|c|}{ Location States Multi } & \multicolumn{2}{|c|}{ Location States Single } & Resources & \multicolumn{2}{|c|}{ Resource States } & Failed Arrivals & Enl \\
\hline & \multicolumn{10}{|c|}{ EJEMPLO SIMULACIÓN.MOD [Normal Run - Rep. 1] } \\
\hline & Name & $\begin{array}{r}\text { Scheduled } \\
\text { Time [HR] }\end{array}$ & Capacity & $\begin{array}{r}\text { Total } \\
\text { Entries }\end{array}$ & $\begin{array}{r}\text { Avg Time } \\
\text { Per Entry } \\
\text { [MIN] }\end{array}$ & $\begin{array}{r}\text { Avg } \\
\text { Contents }\end{array}$ & $\begin{array}{c}\text { Maximum } \\
\text { Contents }\end{array}$ & $\begin{array}{r}\text { Current } \\
\text { Contents }\end{array}$ & \multicolumn{2}{|c|}{ \% Utilization } \\
\hline & BANDA CUELLOS & 10,00 & 30,00 & 50,00 & 200,18 & 16,68 & 27,00 & 27,00 & & 59,58 \\
\hline & BANDA CUERPOS & 10,00 & 30,00 & 50,00 & 200,18 & 16,68 & 27,00 & 27,00 & & 59,58 \\
\hline & PEGADO CUELLOS & 10,00 & 1,00 & 23,00 & 23.11 & 0,89 & 1,00 & 1.00 & & 88,60 \\
\hline & PEGADO BOLSILLOS & 10,00 & 1,00 & 22,00 & 22,73 & 0,83 & 1,00 & 0,00 & & 83,35 \\
\hline & ESTAMPADO & 10,00 & 1,00 & 18,00 & 10,76 & 0,32 & 1,00 & 1,00 & & 32,27 \\
\hline & INSPECCION & 10,00 & 1,00 & 21,00 & 7,68 & 0,27 & 1,00 & 0,00 & & 26,87 \\
\hline & MESA RECHAZOS & 10,00 & 1,00 & 1,00 & 0,00 & 0,00 & 1,00 & 0,00 & & 0,00 \\
\hline & DESCANSO & 8.50 & 1,00 & 0,00 & 0,00 & 0,00 & 0,00 & 0,00 & & 0,00 \\
\hline
\end{tabular}

Figura 3. Ficha de resultados estadísticos de las locaciones involucradas en el proceso. Fuente: ProModel

\section{CASO ISIS PROTEUS}

\subsection{DESCRIPCION DE PROTEUS}

El software utilizado es el programa ISIS PROTEUS intelligent schematic input system (sistema de enrutado de esquemas inteligentes) permite diseñar circuitos electrónicos con componentes variadas desde simples resistencias hasta microcontroladores.

Este software de fácil manejo cuenta con librerías suficientes en las que almacena dispositivos electrónicos y eléctricos para poder realizar simulaciones. 


\subsection{EJEMPLO ISIS PROTEUS}

El siguiente ejemplo muestra como de manera sencilla se puede implementar un contador decimal, con el fin de motivar a los estudiantes a continuar en sus procesos de aprendizaje no dando espera de su aplicación en semestres posteriores sino por el contrario en la medida que van a aprendiendo nuevos conceptos.

En la actualidad es frecuente gracias a la tecnología encontrar publicidad exterior basada en arreglos de leds que muestra slogan, horario o información a los usuarios, esta tecnología es útil pero costosa por este motivo no todas las empresas hacen uso de ella. Pero los costos disminuyen con el tiempo debido a la masificación, pero los costos asociados a la programación y el mantenimiento se conservan.

En la siguiente práctica se muestra un contador de 7 segmentos que nos permite su uso a través de un display para visualizar información digital, para ellos debemos contar con un contador binario BCD de 4 bits en nuestro caso 74ls192 Y un decodificador en nuestro caso 7447 ánodo común.

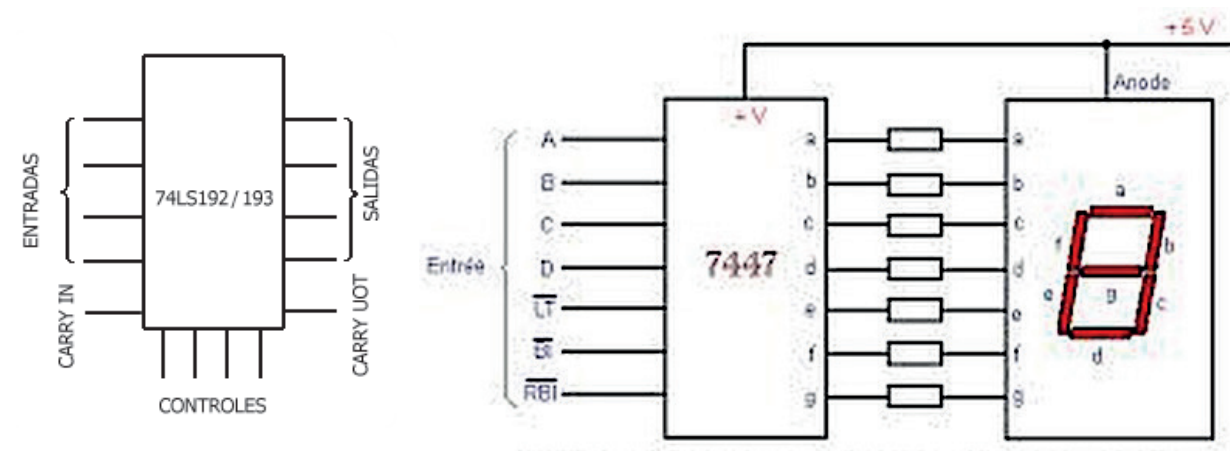

Figura 4. Practica N 1

En el siguiente circuito se muestra este tipo de arreglos, en el arreglo superior se tiene conectado en serie un contador con un decodificador que incrementa sucesivamente sus dígitos y se visualizan en un display.

En el inferior se tiene el mismo arreglo con la diferencia que en el display se visualiza las decenas esto sucede ya que cada vez que se repite una secuencia 0-1-2-3-4-5-6-7-8-9 este

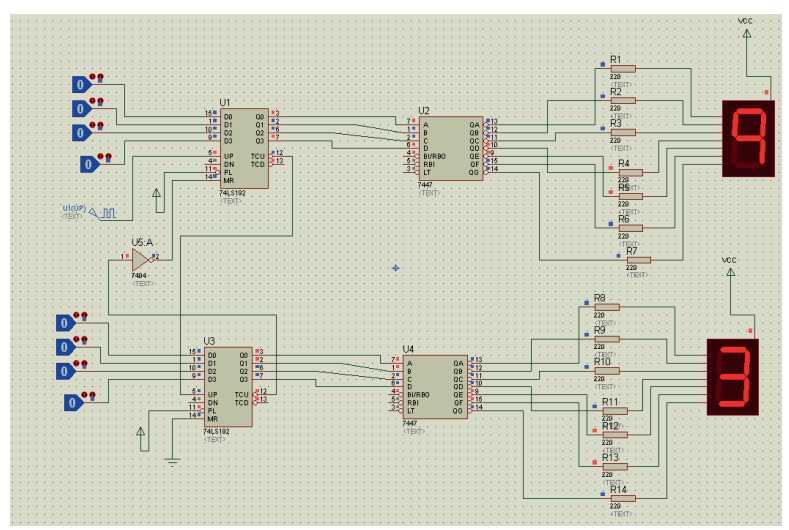

Figura 5. Circuito arreglos envía un pulso al arreglo inferior es decir cada vez que el contador de arriba pasa por el digito 9 el display de abajo se incrementa en una unidad.

En nuestro caso el display va en su tercera iteración a punto de pasar a su cuarta mientras el display muestra el número 9.

Las aplicaciones más comunes son contadores en dispositivos industriales, reloj electrónico, pantallas de exteriores, etc.

\section{CONCLUSIONES}

Este tipo de simulaciones es útil por las siguientes ventajas.

Se verifica lo que se realiza en el laboratorio.

Si no se cuenta con un laboratorio que contenga dispositivos electrónicos en algunas ocasiones costosos y de una vida útil corta debido al manejo de ellos, aun se puede realizar verificaciones de la teoría recibida por los estudiantes. 
El manejo de este tipo de software prepara a los estudiantes para realizar montajes electrónicos mucho más complicados debido a que el programa cuenta con una gran librería útil para otro tipo de montajes.

La ventaja de utilizar simulaciones comparado al realizarlo con dispositivos reales, al menos en los circuitos eléctricos y electrónicos, es casi imperceptible puesto que las perdidas mecánicas son casi nulas algo que no sucede en simulaciones con componentes neumáticas, mecánicas o en situaciones con componentes acoplados mecánicamente.

\section{REFERENCIAS}

[1]. Aguilar, E., \& Viniegra, L. (2003). Atando teoría y práctica en la labor docente. Barcelona: Paidós.

[2]. Colom, Salinas, \& Sureda. (1988). Tecnología y medios educativos. Madrid: Cincel Kapelusz.

[3]. ProModel. (s.f.). Recuperado el 28 de Mayo de 2014, de http://www.promodel.com.mx/ promodel.php

[4]. Spearman, M., \& Hopp, W. (1996). Factory Physics Foundations of Manufacturing Management. Boston: Mc Graw Hill.

[5]. Álvarez, C.(2012). La relaciónteoría-práctica en los procesos de enseñanzaaprendizaje. Educatio Siglo XXI, vol. 30, n² 2, 383-402. Recuperado de http://revistas.um.es/ educatio/article/view/160871

[6]. Ávalos, B. (2009). Los conocimientos y las competencias que subyacen a la tarea docente. En C. Vélaz de Medrano y D. Vaillant (Coord.), Aprendizaje y desarrollo profesional docente (67-78). Madrid: Fundación Santillana-OEI

[7]. Brouwer, N., y Korthagen, F. (2005). Can teacher education make a difference? American Educational Research Journal, vol. 42, $n^{\circ} 1,153-224$.

[8]. Edmonds, E. y Candy, L. (2010). Relating theory, practice and evaluation in practitioner research. Leonardo, vol. 43, $n^{\circ}$ 5, 470-476. Recuperado de http://www. upv.es/laboluz/master/metodologia/textos/ Theory_practice_evaluation.pdf

[9]. Feiman Nemser, S. (1990). Teacher's preparation: structural and conceptual alternatives. En W.R. Houston (Ed.), Handbook of research on teacher education (212-233). Nueva York: Macmillan

[10]. Feiman Nemser, S.; Buchmann, M.; Ball, D. (1986). Constructing knowledge about teaching: research in progress on beginning elementary teachers. San Francisco: Paper AERA

[11]. Flores, M.A. y Day, C. (2006) Contexts which shape and reshape new teachers' identities: A multi-perspective study. Teaching and Teacher Education, $n^{\circ}$ 22, 219-232.

[12]. Flores, M.A. (2000). A Inducao no ensino: desafios e constrangimentos. Lisboa: Instituto de Inovacao Educacional.

[13]. Geertz, C. (1987). La interpretación de las culturas. Barcelona: Gedisa.

[14]. Gil Cantero, F. (2011). Educación con teoría: revisión pedagógica de las relaciones entre la teoría y la práctica educativa. Revista Teoría de la Educación, vol. 23, no 1, 1943. Recuperado de http://campus.usal.es / revistas_trabajo/index.php/ 11303743/ article/viewFile/8575/8809

[15]. Gimeno, J. (1998). Poderes inestables en educación. Madrid: Morata.

[16]. Glaser, B. y Strauss, A. (1967). The discovery of grounded theory: strategies for qualitative research. New York: Aldine de Gruyter.

[17]. González-Sanmamed, M. y FuentesAbeledo, E.J. (2010). El Practicum en el aprendizaje de la profesión docente. Revista de Educación, n 354, 47-70. Recuperado de http://www.revistaeducacion.educacion. es/re354/re354_03.pdf

[18]. Imbernón, F. y Colén, M.T. (2015). Los vaivenes de la formación inicial del profesorado. Una Reforma siempre inacabada. Tendencias Pedagógicas, $n^{\circ}$ 25, 57-76. Recuperado de https:// dialnet.unirioja.es/servlet/articulo? codigo $=5236973$ 